\title{
Cognitive schemas: how can we use them to improve children's acceptance of diverse and unfamiliar foods?
}

\author{
Patricia Pliner* \\ Department of Psychology, University of Toronto at Mississauga, 3359 Mississauga Rd, Mississauga, ON L5L 1C6, Canada
}

Foods represent important stimuli for humans, especially for human children. After weaning, it is important that children quickly acquire knowledge about their food environment to avoid ingesting potentially dangerous substances. This paper discusses this process and its implications in terms of schemas. The effects of providing positive taste information to novel foods and of adding familiar flavors to novel foods are interpreted by means of the schema construct. A means of changing schemas through exposure to schema-inconsistent information is presented and evidence for its efficacy is described. Finally, the effect of early variety on subsequent willingness to eat unfamiliar foods is described and once again interpreted by means of the schema construct.

Novel foods: Dietary variety: Children: Social cognition: Schema: Food neophobia

Foods represent important stimuli for humans, especially for human children. After weaning, it is important that children quickly acquire knowledge about their food environment to avoid ingesting potentially dangerous substances. This paper discusses this process and its implications in terms of schemas, a construct that social psychologists use to talk about people's knowledge about the stimuli in their environments ${ }^{(1)}$. Schemas are cognitive structures that represent organized knowledge about a given type or category of stimulus. Individuals have schemas about all kinds of stimuli. Social psychologists are most interested in social schemas, those referring to people (e.g., university professors, Englishmen, women); however, schemas can also be about other concepts (e.g., furniture), or even about events (e.g., meals). Included in the content of a schema are individuals' basic knowledge and impressions - their ideas about the features that characterize stimuli in that particular category. For example, one's schema about university professors might include the information that they are bookish, methodical, verbose, and badly-dressed. A meal schema might include the notions that a meal involves taking of food while sitting down, the use of dishes and utensils, heating of the food, a particular order of courses ${ }^{(2,3)}$. There is considerable research indicating that both children and adults use schemas in processing information and that such schemas can affect reactions to previously unencountered members of a category of stimuli. If one meets a new university professor, his or her schema leads to particular expectations about what this person is going to be like, and these expectations are likely to affect one's behavior toward this person. In this paper, we explore the implications of the idea that people have schemas about novel foods.

In order to understand the content of children's schemas about novel foods, we offered them a series of such foods ${ }^{(4)}$.
Then we questioned the children about the foods they had rejected, asking "How come you didn't want to taste the ?" The children's responses revealed negative evaluations of these foods, based on their appearance ("it looks yucky," "it looks like liver," "it looks like barf," "it looks rotten") and negative expectations about their taste ("I don't like it," "I won't like it," "I don't like vegetables"). In other studies, when we have asked children to rate how much they think they would like novel foods, the ratings are always lower than those for familiar foods ${ }^{(5)}$. We have also conducted studies with adults who also have a similarly negative view of the taste of novel foods. Further, they consider them slightly dangerous and rate themselves as somewhat anxious at the prospect of eating them ${ }^{(6)}$. Thus, people's schemas about novel foods include the information that they are unpalatable, unappealing, not likeable and even slightly dangerous. It is a fairly simple schema, yet a schema nonetheless.

And it is these negative expectations about novel foods that makes people avoid them. It is as though people have a default expectation: "if it's novel, it won't taste good, and if it's not going to taste good, I don't want to eat it." And that reaction is what is referred to as food neophobia. It has been argued that this neophobia is adaptive and serves a protective function in a potentially hostile food environment ${ }^{(7)}$. Cashdan $^{(8)}$ has shown that neophobia is at its strongest just at the time when the developing child would be most vulnerable to this dangerous environment, around the age of two, when in traditional cultures the child is weaned and therefore ingesting foods other than mother's milk and also at the time the child becomes relatively mobile and likely to stray from adult supervision. And, further, it occurs before the child has the cognitive resources to understand verbal instructions 
about not eating strange berries and things he or she picks up on the street. So, the child is protected to some degree by its neophobic reaction to unfamiliar foods. For parents and from a nutritional standpoint that can be a problem because there are many novel foods that are not harmful and that the child would benefit from eating. But the child seems to be biased against them.

This bias is not surprising. A recent article on cognitive biases in general suggests that such biases might reflect the survival value or relative costs of false positives and false negatives ${ }^{(9)}$. A false positive occurs when a person does something although it does not produce the anticipated benefit, while a false negative occurs when a person fails to do something that, if done, would have produced a benefit. Each might ultimately have negative consequences, but the magnitudes of the negative consequences are often very unequal. Hazard detection is biased toward "false alarms." For example, it is better to have one's smoke detector go off too often than not often enough. Whenever costs are asymmetrical, people are biased toward making the least costly error. In the case of novel foods, the cost of a false negative (failing to avoid something that shouldn't be eaten) is probably greater than the cost of a false positive (avoiding something that should be eaten). In the first case, the outcome could be catastrophic -the individual could die. In the second, the outcome is much less so - the individual might miss out on a nutritious food. As a result, people/children are biased toward avoiding novel foods. They are overly cautious in order to avoid a costly false negative. In a sense, their schemas about novel foods are instantiations of this bias.

So, assuming that children's negative bias toward novel foods is reflected in their schemas and their schemas maintain this bias, how can we use that information to get children to try novel foods? One strategy would be to persuade them that in a particular case, the schema does not apply (see panels $a$ and $b$ of Fig. 1). In other words, the set of characteristics contained in the schema are not true of this food. This food is an exception: it is an atypical member of its category. This novel food is not yucky, doesn't taste bad, is not dangerous - it's good. In fact, that's one of the things that parents do instinctively when they want to get their children to try something new. "Try it - you'll like it. It's good."

There are many studies showing that simply telling people that a novel food tastes good or tastes like something else that tastes good ("it tastes just like chicken"), increases their willingness to try it. For example, Pelchat \& Pliner ${ }^{(10)}$ looked at the effect of this kind of "good taste" information on willingness to try novel foods in a school cafeteria in which a meal was pre-paid; students could take whatever they wanted as they went through the line. Pairs of experimental foods were presented simultaneously. In each case, one was novel and the other, familiar (e.g., tortilla chips and blue corn tortilla chips). In experimental conditions, the novel food was accompanied by a sign with "good taste" information; in the control condition, there was no sign. Experimenters, disguised as cafeteria workers, looked to see whether person took the familiar food, the novel food, or both. There was a clear effect of taste information on choice of novel food; in the taste information condition, $55 \%$ of the children took a novel food while in the control condition, only $39 \%$ did so.

Sometimes, especially with younger children, researchers (and parents) present the information that a novel food tastes good by exposing the child to someone (a "model") who eats the food. Instead of telling the children the food tastes good, they show them it tastes good. There are many
(A)
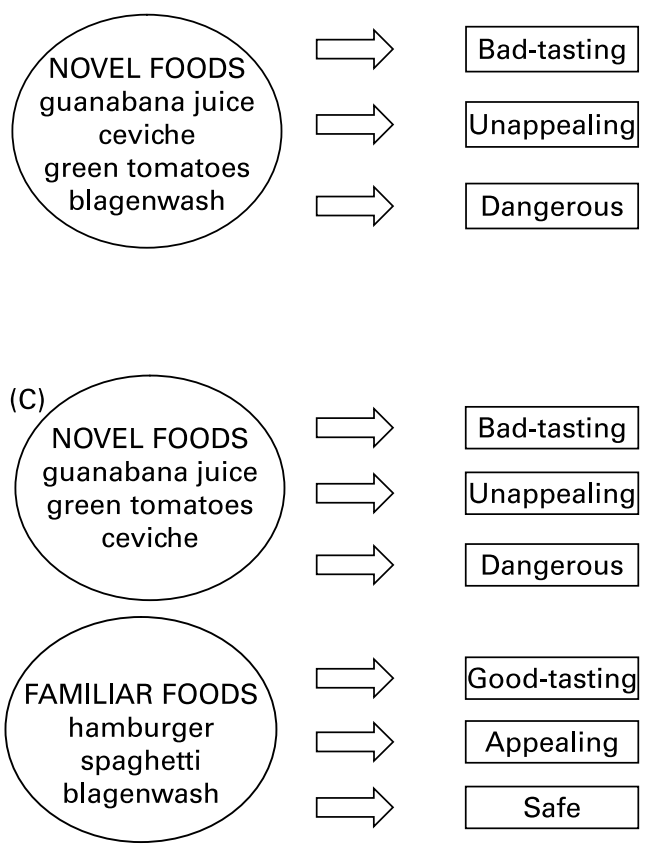

(B)

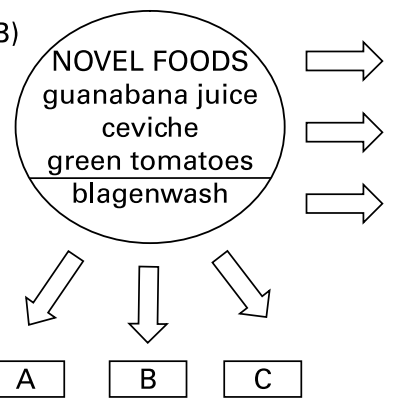

(D)
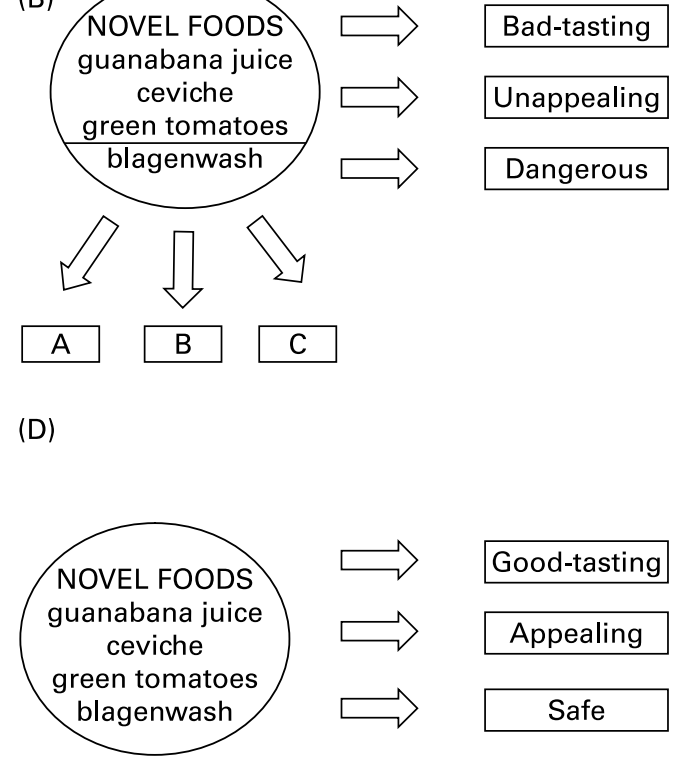

Fig. 1. (A) Representation of a novel food schema, (B) A food for which the novel food schema does not apply, (C) Moving a novel food to the familiar food category, (D) Changing the content of a novel food schema. 
studies showing that children are more likely to eat a novel food in the presence of a model who has done so ${ }^{(11-13)}$. A recent study ${ }^{(14)}$ shows that it must be the same food - if someone is eating a different novel food, it does not elicit modeling. Other studies have shown that children are more likely to copy a model when it is their mother rather than a stranger ${ }^{(15)}$ and when the model expresses liking for the food ${ }^{(16)}$. In the schema context, what these manipulations are doing is telling the individual that the (bad taste) information contained in the novel food schema does not apply to this food - it is an exception.

Another way of circumventing people's novel food schemas is to persuade them that a particular food isn't novel - it doesn't belong in the novel food category (see panel c of Fig. 1). Here Elisabeth Rozin's notion of "flavor principles" is relevant ${ }^{(17)}$. Flavor principles are the distinctive and pervasive seasoning combinations that characterize and even define many cuisines. For example, what makes southern Italian food seem "Italian" is the fact that it contains olive oil, tomato, and basil or oregano. What makes Chinese food seem "Chinese" is the fact that it is flavored with soy, rice wine, and ginger. Within a culture, flavor principles are ubiquitous, appearing in many dishes and appearing many times a day; thus they are very familiar, and very much liked. E. Rozin \& P. Rozin ${ }^{(18)}$ note that individuals have a deep attachment to their culture's characteristic seasonings and find it difficult to imagine food prepared without them. The Rozins have suggested that one of the functions of flavor principles is to facilitate the introduction of a new food staple into a culture; that is, adding the familiar flavor principle to the new food increases willingness to try it by making it appear more familiar (and likely to taste good).

Stallberg-White \& Pliner ${ }^{(19,20)}$ demonstrated this phenomenon in two studies designed as laboratory analogues of the introduction of a new food into a culture. They offered participants some novel foods to taste; some were offered in combination with familiar flavour principles in the form of sauces and condiments tailored to the specific experiences of each participant while others were offered with no sauce or condiment. They found that the addition of familiar flavour principles to novel foods increased the willingness of both adults and children to taste them (see Fig. 2). From the schema perspective this kind of strategy involves moving food out of novel food category by adding a familiar flavor principle.

Thus far, we have described two schema-based techniques for getting people to try unfamiliar foods. One involves convincing them that a particular food is an unusual or

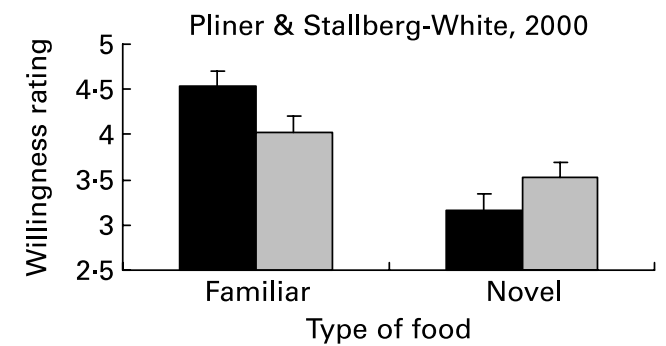

Fig. 2. Adding a familiar flavor principle to a novel food increases willingness to eat it. atypical member of the category of unfamiliar foods; it does not share the usual attributes. The other is to convince them that the food in question does not belong in the category of unfamiliar foods at all.

However, if the goal is to increase willingness to taste novel foods in general, then neither of these techniques is very efficient because they focus on particular foods - they do not extend beyond the particular food in question. Instead of introducing a long series of exceptions, it would be more efficient to change the content of the novel food schema. The goal would be to change the schema to include the information that novel foods are palatable, likeable, and generally desirable. Research in social psychology has shown that it is possible to change people's schemas by presenting them with schema-inconsistent information. In other words, one provides many exemplars which challenge the existing view of the stimuli in a particular category.

In the case of novel foods, provision of such schema-inconsistent information would involve taste exposure to good-tasting novel foods. If an individual thinks that novel foods taste bad, then a good-tasting novel food is inconsistent with his or her schema. In two studies by Pliner \& Loewen ${ }^{(5)}$, some children received schema-inconsistent information in form of exposure to a set of four novel foods that had been carefully pretested to ensure that they were extremely palatable. Other children received information irrelevant to novel food schema in form of exposure to a similar set of palatable familiar foods. Such exposure would not provide any information about novel foods. Finally, a third group of children received exposure to a set of unpalatable novel foods; such foods would be consistent with a schema containing the information that novel foods taste bad. In the second part of the study, the children were given a behavioral neophobia choice task; they had to choose which foods they would taste later in the session from a series of both novel and familiar foods. These foods were different from the ones in the exposure part of the study; no child had seen these foods earlier in the study. Children in the first group, those receiving pre-exposure to good-tasting, schemainconsistent foods, accepted more of the novel foods in the subsequent behavioral neophobia task than did those in either of the other two groups. Similar results were obtained in a study with adult participants ${ }^{(6)}$. We interpreted these data to suggest that we managed to change people's schemas to include the idea that novel foods taste good; because their schemas changed, they became more willing to try novel foods.

To this point, the discussion has focused on changing children's schemas about novel foods. It is also possible that if one can intervene early enough, one can encourage them to form schemas in the first place that include the information that novel foods are good and fun to eat and basically no different from familiar foods. It might be possible to do that by giving them a great deal of exposure to a variety of foods at an early age. The foods will be novel because, until young children had a large amount of experience with foods, most foods are novel. Given the schema framework, it is important that the foods, particularly those presented earliest, be relatively palatable in order to induce an appropriate schema (containing the information that novel foods taste good).

There is a reasonable amount of correlational evidence that supports this idea. That is, children who are exposed at an early age to a large amount of variety (which, once again, 
would of necessity include novel foods) are more accepting of new, different novel foods when they are older. Pelchat \& Pliner ${ }^{(21)}$ found that mothers' reports of their 2-6 year old children's current willingness to eat novel foods was related to their reports of early variety in the child's diet. More recently, Russell ${ }^{(22)}$ examined many aspects of parental attitudes and beliefs, feeding practices and their children's food-related attitudes and behaviors. She found that of the four significant predictors of children's food neophobia, three had to do with offering variety: taking the child to restaurants serving unfamiliar foods, offering the child untried foods, exposing the child to variety. Skinner et al. ${ }^{(23)}$ showed that exposure/variety between 2-24 months of age was a significant predictor of children's acceptance of fruits at 6-8 years. Rigal and colleagues ${ }^{(24)}$ studied a group of children who formerly had food allergies but who had outgrown them; as part of management of the allergies they were required to eat restricted diet during early childhood. These children were more food neophobic than their nonallergic siblings. Within the allergy group, there were positive correlations between the number of foods forbidden by the physician and also the number of foods excluded by the mother and a measure of neophobia.

In sum, early variety is positively related to later acceptance of novel foods. These data are suggestive but not totally convincing. First, all are based on mothers' reports (mostly retrospective) and, therefore, subject to various biases. Further, all are correlational, subject to the usual ambiguity of direction of causality with that method. A recent study by Gerrish \& Menella $^{(25)}$ uses the experimental method, thereby circumventing many of these problems. On Day 1, 4.5 month-old infants who had not yet been exposed to solid foods were tested in the laboratory for acceptance of carrots. On Days $2-10$, in addition to their usual diets, they were fed at home by their mothers one serving per day of one of the following: 1) carrots; 2) potatoes; or 3) a variety regimen of peas, potatoes, and squash, one per day in sequence, the sequence repeated three times. On Day 11 they were tested in the laboratory for acceptance of carrots. Babies in Groups 1 and 3 showed a greater increase in acceptance of carrots than did those in Group 2 (see Fig. 3). On Day 12, they were tested in the laboratory for acceptance of chicken. Babies in Group 3 were more accepting of the chicken than those in the other two groups. Thus, the babies with the greatest manipulated exposure to variety were subsequently more willing to

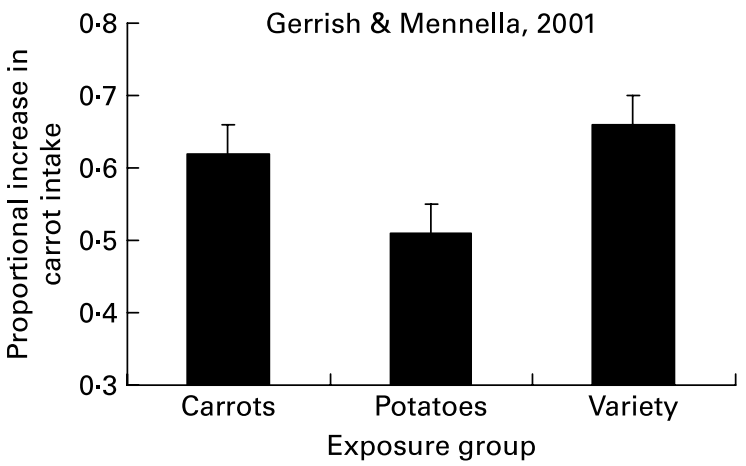

Fig. 3. Exposure to either carrots or a variety of vegetables, not including carrots, increases subsequent consumption of carrots. accept novel foods, an impressive experimental demonstration of the effects of early exposure to variety.

We find the schema framework to be a useful heuristic for understanding the literature on children's food neophobia. When one conceptualizes children's reactions to novel foods as schema-influenced responses, it is easy to understand how and why various strategies for increasing willingness to eat specific novel foods are successful. It also suggests a more general approach to the problem of increasing children's acceptance of novel foods. Although the schema framework was applied post hoc to the existing literature, it also makes a prediction not previously tested in research. That is, to the extent that early exposure to variety provides a means for inducing a positive rather than a negative schema about novel foods, it should be important that the earliest foods presented be high in palatability. Schemas are based in large measure on the individual's experiences with stimuli in a particular category. Negative experiences produce a schema with negative content, while positive experiences produce a schema with positive content. Furthermore, once formed, schemas are relatively impervious to inconsistent information; that is, it would take a number of instances of schema-inconsistent information to change a schema. Thus, it would be predicted that if a child were presented with unpalatable novel foods early in the formation of his or her schema, that schema should not be positive. Thus, it would be important to ensure that the earliest novel foods to which the child is exposed are relatively palatable. Davis' ${ }^{(26)}$ research on selfselection diets suggests that young children find a wide range of foods to be palatable, including notably fruits and cereals. Indeed, for the three infants whose diets formed the basis of her first report on the self-selection paradigm, fruit constituted from 15 to 50 percent of their total intake by weight over a six-month period. So, practically speaking, infants' introduction to solid foods should begin with those that are most likely to be liked - perhaps fruits, given Davis' results and given that we know from other sources that sweet foods are innately and universally palatable. Note that the suggestion here is not that children's early exposure be to the kinds of high fat sweet foods so beloved of older children and so deplored by nutritionists. The suggestion is that parents capitalize on children's natural liking for the taste of fruit to help promote the formation of positive novel food schemas. Although such a suggestion has never been tested, either experimentally or correlationally, it should be possible to do so. Positive results would provide support for the schema framework described in this paper.

\section{Conflict of interest statement}

The research reported in this paper was supported by the Social Sciences and Humanities Research Council of Canada. The author has no conflicts of interest to report.

\section{References}

1. Fiske ST \& Taylor SE (1991) Social Cognition, 2nd ed. New York: McGraw-Hill, Inc.

2. Pliner P \& Martins Y (2002) The effects of meal cues and amount consumed on predictions of future eating behavior. Pers Soc Psychol Bull 28, 1354-1365. 
3. Pliner P \& Zec D (2007) Meal schemas during a preload decrease subsequent eating. Appetite 48, 278-288.

4. Pliner P (1994) Development of measures of food neophobia in children. Appetite 23, 147-163.

5. Pliner P \& Loewen R (1999) Effects of prior exposure to palatable and unpalatable novel foods on children's willingness to taste other novel foods. Appetite 32, 351-366.

6. Pliner P, Pelchat M \& Grabski M (1993) Reduction of neophobia in humans by exposure to novel foods. Appetite 20, $111-123$.

7. Pliner P \& Hobden K (1992) Development of a scale to measure the trait of food neophobia in humans. Appetite 19, $105-120$

8. Cashdan E (1994) A sensitive period for learning about food. Hum Nat 5, 279-291.

9. Haselton MG \& Nettle D (2006) The paranoid optimist: an integrative evolutionary model of cognitive biases. Pers Soc Psychol Rev 10, 47-66.

10. Pelchat P \& Pliner P (1995) "Try it: you'll like it": effects of information on willingness to try novel foods. Appetite 24, 153-165.

11. Birch LL (1980) Effects of peer model's food choices and eating behaviors on preschooler's food preferences. Child Dev 51, 489-496.

12. Duncker K (1938) Experimental modification of children's food preferences through social suggestion. J Abnorm Soc Psychol 33, 489-507.

13. Marinho H (1940) Social influence in the formation of enduring preferences. J Abnorm Soc Psychol 37, 448-468.

14. Addessi E, Galloway A, Visalberghi E \& Birch LL (2005) Specific social influences on the acceptance of novel foods in 2-5-year-old children. Appetite 45, 264-271.
15. Harper LV \& Sanders KM (1975) The effects of adults' eating on young children's acceptance of unfamiliar foods. J Exp Child Psychol 20, 206-214.

16. Hendy HM \& Raudenbush B (2000) Effectiveness of teacher modeling to encourage food acceptance in preschool children. Appetite 34, 61-76.

17. Rozin E (1973) The flavor principle cookbook. New York: Hawthorne Books.

18. Rozin E \& Rozin P (1981) Culinary themes and variations. Nat Hist 90, 6-14.

19. Stallberg-White C \& Pliner P (1999) The effect of flavor principles on willingness to taste novel foods. Appetite 33, 209-221.

20. Pliner P \& Stallberg-White C (2000) "Pass the ketchup, please": familiar flavors increase children's willingness to taste novel foods. Appetite 34, 95-103.

21. Pelchat M \& Pliner P (1986) Antecedents and correlates of feeding problems in young children. J Nutr Educ 18, 23-29.

22. Russell CG (2006) Parental socialization of preschool children's food preferences. Unpublished Ph.D. Thesis, Deakin University.

23. Skinner JD, Carruth BR, Bounds W, Ziegler P \& Reidy K (2002) Do food-related experiences in the first 2 years predict dietary variety in school-aged children? J Nutr Educ Behav 34, 310-315.

24. Rigal N, Reiter F, Morice C, Boissieu DD \& Dupont C (2005) Food allergy in the child: an exploratory study on the impact of the elimination diet on food neophobia. Arch Pediatr 12, 1714-1720.

25. Gerrish CJ \& Menella JA (2001) Flavor variety enhances food acceptance in formula-fed infants. Am J Clin Nutr 73, $1080-1085$.

26. Davis CM (1928) Self selection of diet by newly weaned infants: an experimental study. Am J Dis Child 36, 651-679. 